\title{
A Study on Staircase Detection for Visually Impaired Person by Machine Learning using RGB-D Images
}

\author{
Manabu Shimakawa*, Runa Akutagawa, Kimiyasu Kiyota, Mitsutaka Nakano \\ National Institute of Technology, Kumamoto College, 2659-2 Suya, Koshi, Kumamoto 861-1102, Japan \\ *Corresponding Author: shimakawa@ @umamoto-nct.ac.jp
}

\begin{abstract}
There are some previous researches and developments which have purposes to support for visually impaired person. In facts, a survey report about accidents of visually impaired pedestrian said that $42 \%$ of respondents experienced walking accidents. Our previous studies had taken an approach of using some image processing techniques to detect the steps of staircases on depth images taken by RGB-D camera. To improve the detection accuracy, this paper applied machine learning techniques, e.g. HOG (Histogram of Oriented Gradients) feature descriptor and AdaBoost classifier, to the staircase detection. This paper shows experimental results of the staircase detection applied to depth-images and RGBimages, and mentions about the effectiveness. Moreover, the classifier AdaBoost and SVM (Support Vector Machine) were compared by a viewpoint about detection accuracy.
\end{abstract}

Keywords: Staircase Detection, Visually Impaired, Machine Learning, RGB-D Image, AdaBoost, SVM, HOG.

\section{Introduction}

According to the WHO fact sheets ${ }^{(1)}, 285$ million people were estimated to be visually impaired worldwide: 39 million were blind and 246 have low vision. Perhaps the number of people is continuing to increase. The visually impaired has a high need to go out, however there are so many dangers. In facts, a survey report ${ }^{(2)}$ about accidents of visually impaired pedestrian said that $42 \%$ of respondents experienced accidents during walking.

Research and development of support tools for the walking of visually impaired people have been done for a long time. The smart electronic white cane ${ }^{(3)}$ is one of walking support devices commercialized. It has ultra-sonic sensors, alerts the danger with vibration when it detects obstacles above and/or front position. There is also research $^{(4)}$ to detect staircase and pedestrian traffic signals using images of RGB-D camera. Our previous studies ${ }^{(5,6)}$ had taken an approach of using some image processing techniques to detect the steps of staircases on depth images by RGB-D camera. However, the RGB-D camera is large and heavy, it is difficult to handle, so we aim to implement it on smartphone with RGB camera ${ }^{(7)}$.

This paper describes about staircase detection by using image processing techniques and machine learning approach, and compares with AdaBoost and SVM as classifier algorithm by a viewpoint about detection accuracy with some experimental results.

\section{Detection by Image Processing Techniques}

\subsection{RGB-D Camera}

The camera that can take RGB images and depth images is called RGB-D camera. Microsoft Kinect is well known as a typical device. Fig. 1 shows examples of RGB and depth images taken by Microsoft Kinect. Each pixel of the depth image expresses a distance from the camera. It is white as the distance is closer, and it is blacker as distant. Both images consist of $640 \times 480$ pixels.

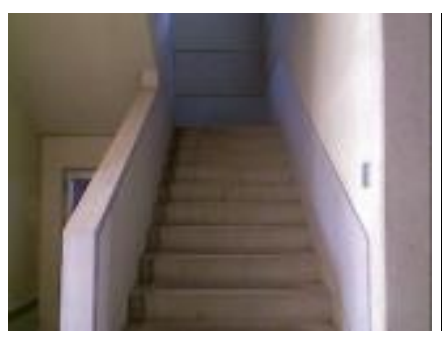

(a) RGB image

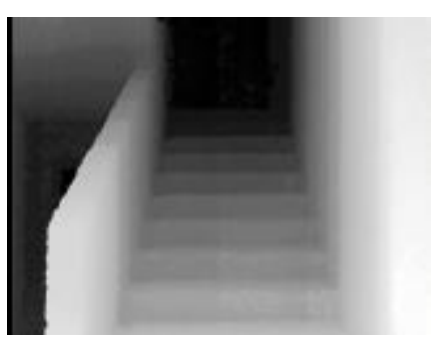

(b) Depth image
Fig. 1. Images taken by Microsoft Kinect. 


\subsection{Staircase Detection Procedure}

The staircase detection procedure by image process techniques is as follows:

(Step 1) Taking an input depth image.

(Step 2) Edge detection with Canny method (Fig.2(a)).

(Step 3) Extraction of line segments with Hough transform.

(Step 4) Selection of step candidates from extracted line segments (Fig.2(b)) with the following criteria:

- The line segment should be almost horizontal, and it needs sufficient length to some extent.

- Depth difference of each neighbor two line segments should be within $200-350 \mathrm{~mm}$.

- The distance of a line segment above should be farther than below one.

(Step 5) Identification of staircase area (Fig.2(c)).

\subsection{Experimental results}

This method was applied in several staircase situations. Fig.2(c) shows a success situation to detect staircase area. However, like in Fig.2(d), staircase detection may not be successful when a person partially hides the stairs. Since this method requires that the line segment of the step candidate has sufficient length to some extent, when the line segment becomes shorter due to shielding, it is not recognized as a staircase area.

\section{Machine Learning for Depth Images}

\subsection{Machine Learning Algorithm}

This paper used the traincascade tool for machine learning in OpenCV. The traincascade is a machine learning tool to create a cascade classifier for object detection, implements AdaBoost as a machine learning algorithm and it supports feature descriptors of HOG, Haar, and LBP. Here, this study selected HOG feature descriptor that is widely used for object detection.

\subsection{Experimental Results}

This experiment employed 57 positive images and 30 negative images as training data. The number of the images is too small, but it is enough to check the trend of the cascade classifier ability. Fig. 3 shows examples of depth images that were used for machine learning. The experimental results are shown in Fig. 4. Rectangles with red colored lines indicate detected staircase areas. As shown in Fig.4(a), good results were obtained in stairs where there is

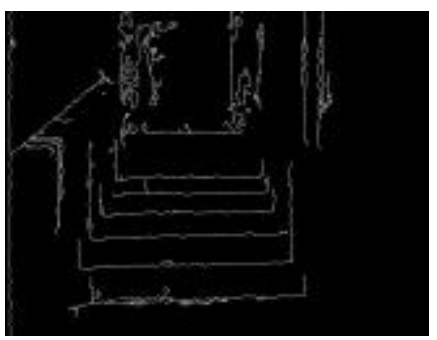

(a) Edge detection

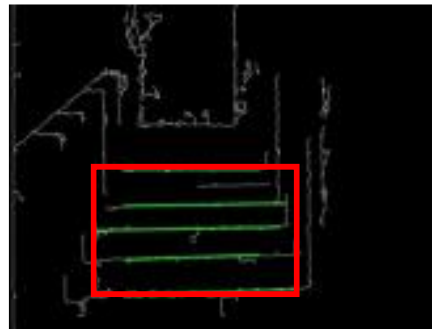

(c) Staircase area (b) Step candidates

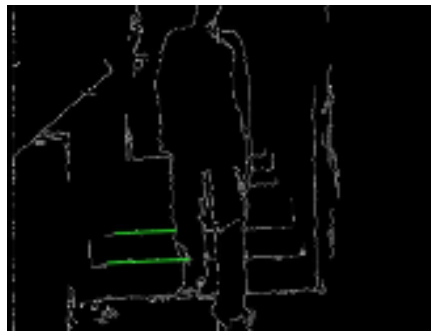

(d) Failure case

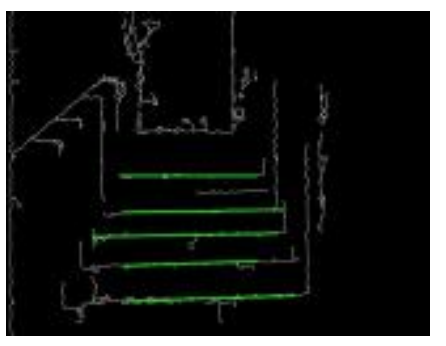

Fig. 2. Detection procedure by image processing techniques and its experimental results.

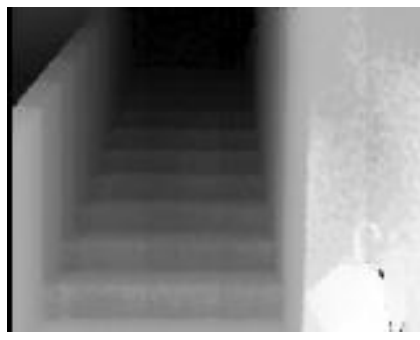

(a) Positive image

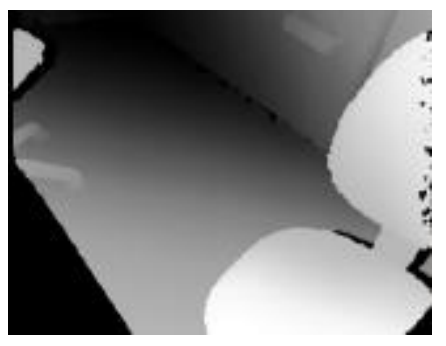

(b) Negative image
Fig. 3. Examples of depth images for machine learning.

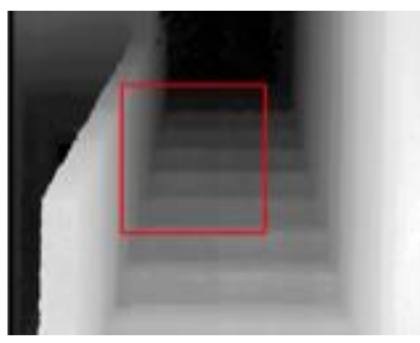

(a) Case with no person

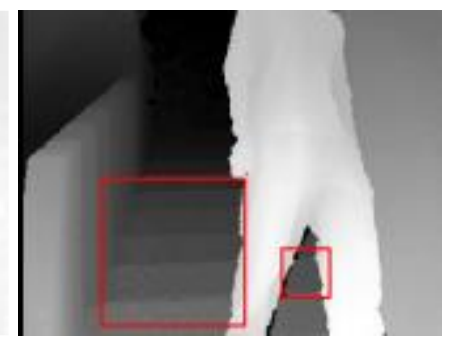

(b) Case with a person
Fig. 4. Experimental results of machine learning with depth images.

nothing to block. Even if a person hides stairs partially, the remained stairs were detected well, Fig. 4(b).

\section{Machine Learning for RGB Images}

\subsection{Concept}

Experiments in the previous section revealed that the 
depth image is convenient for staircase detection. However, the RGB-D camera like a Microsoft Kinect is large and heavy, it is difficult to handle, so such camera sensor device should be replaced to smartphone or portable device with RGB camera to implement it on. In this section, machine learning algorithm is applied to RGB image aiming to smartphone implementation.

\subsection{Machine Learning Algorithm}

The traincascade tool in OpenCV is also used to machine learning for RGB images as same as for depth images, employs AdaBoost as a machine learning algorithm and HOG feature descriptor.

\subsection{Experimental Results}

Experiments were conducted to verify the effectiveness of machine learning for staircase detection using RGB images. This experiment employed 250 positive images and 200 negative images for training.

The experimental results are shown in Fig. 5. Red colored rectangles indicate detected staircase areas. Fig.5(a) is a case of no person blocks the stairs, the rectangle indicates almost all staircase area. On the other hand, Fig.5(b) shows a case where a person hides partially the staircase, a good result is obtained also in such cases. However, in this experiment, the accuracy of this machine learning method to the staircase detection has not been verified.

\section{Comparison with AdaBoost and SVM}

\subsection{Machine Learning Algorithms}

Both AdaBoost (Adaptive Boosting) and SVM (Support Vector Machine) are machine learning algorithms used as classifiers. AdaBoost uses multiple weak classifiers with low performance to consist a strong classifier with high performance. The advantages of AdaBoost are

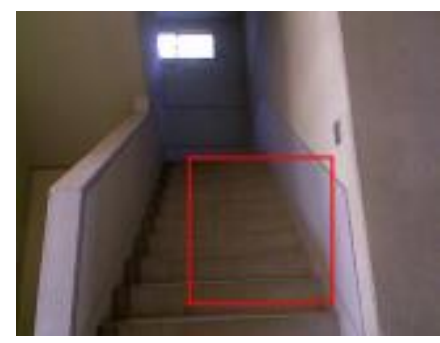

(a) Case with no person

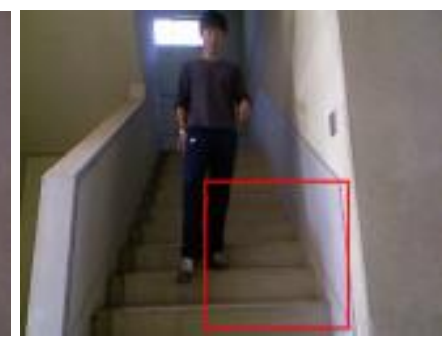

(b) Case with a person
Fig. 5. Experimental results of machine learning with RGB images. simple calculation, fast, and tuning is not necessary.

SVM is one of the pattern recognition algorithms. A support vector machine constructs a hyperplane in a highdimensional space to separate into two classes. A good separation is achieved by the hyperplane that has the largest distance to the nearest training-data point of any class, since in general the larger the margin the lower the generalization error of the classifier.

This paper compares the performance of AdaBoost and SVM as classifier for staircase detection. Both machine learning algorithms used HOG feature descriptor.

\subsection{Experimental Results}

This experiment also employed 250 positive images and 200 negative images for training as same as section 4.3. Fig. 6 shows experimental results of AdaBoost and HOG feature description, and Fig. 7 shows in case of SVM and HOG combination. To make it easy to compare the detection performance of them, divide each image into small areas of $25 \times 25$ pixels.

As shown in Fig.6(a) and Fig.7(a), both algorithms AdaBoost $+\mathrm{HOG}$ and $\mathrm{SVM}+\mathrm{HOG}$ can classify staircase almost well. There is no big difference, but SVM+HOG is better a little. However, in the landscape image of street where no staircase as shown in Fig.6(b) and Fig.7(b), SVM+HOG detected some area as stairs erroneously more than AdaBoost+HOG.

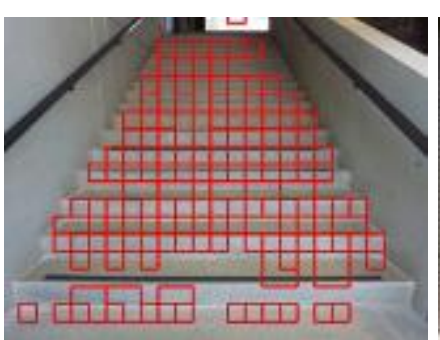

(a) Staircase

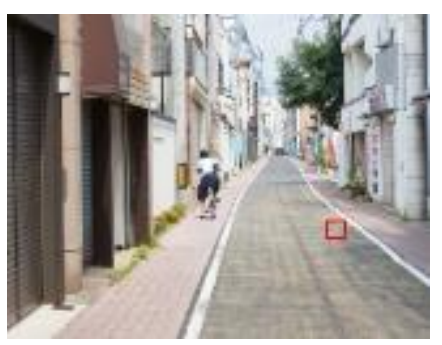

(b) Street
Fig. 6. Experimental results of AdaBoost $+\mathrm{HOG}$.

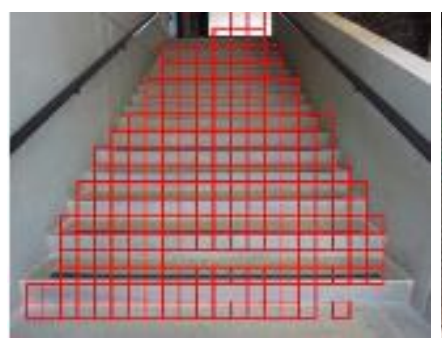

(a) Staircase

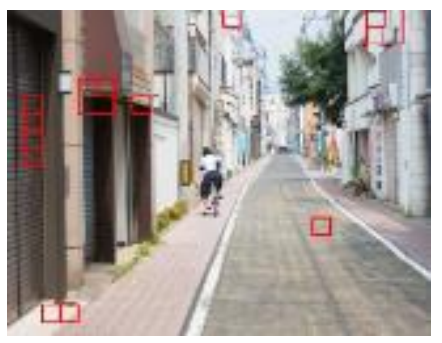

(b) Street
Fig. 7. Experimental results of SVM $+\mathrm{HOG}$. 


\subsection{Experimental Results with Additional Condition}

Since the detection target is a staircase, it is considered that the identified small areas are adjacent in the horizontal direction. Therefore, to reduce error detections, add the following condition:

- Four or more small areas in adjacent 8 neighborhoods should be identified as staircase.

The results with the additional condition are shown in Fig. 8 and Fig. 9. According to Fig.8(b) and Fig.9(b), it was possible to reduce error detections in both methods.

\subsection{Performance Evaluation with ROC curve}

Receiver Operating Characteristic (ROC) curve was used for performance evaluation of both machine learning algorithms. The area under the graph takes a value from 0 to 1 , indicating that the closer the value is to 1 , the higher the classification ability. From Fig. 10, in this case, it is understood that AdaBoost has better performance than SVM.

\section{Conclusions}

In this paper, the machine learning approaches performed better classification ability about staircase detection than using conventional image processing techniques. It is found from some experiments that the approaches were effective not only for depth images but also

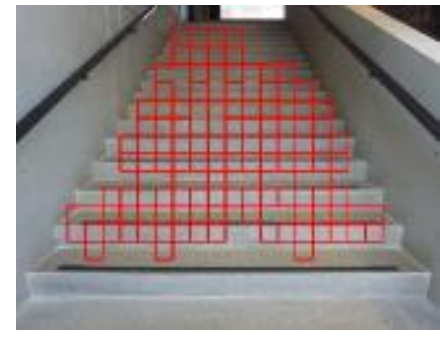

(a) Staircase

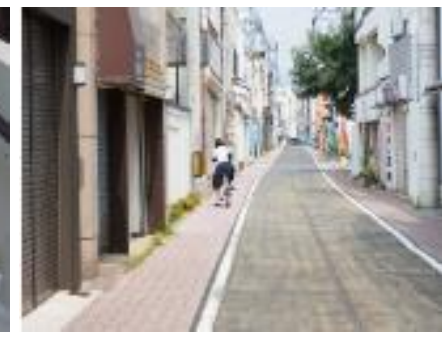

(b) Street
Fig. 8. Experimental results of AdaBoost $+\mathrm{HOG}$ with additional condition.

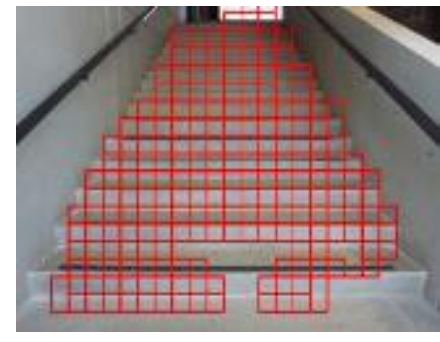

(a) Staircase

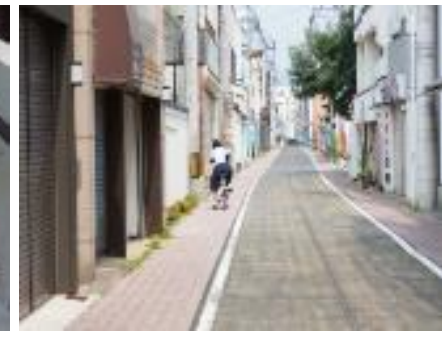

(b) Street
Fig. 9. Experimental results of SVM $+\mathrm{HOG}$ with additional condition.

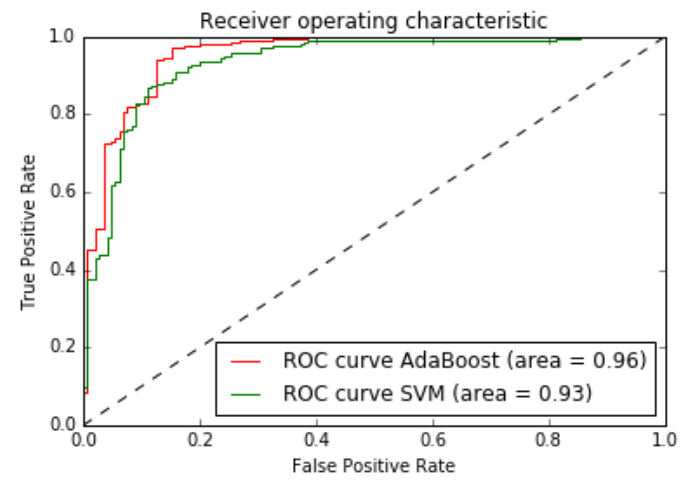

Fig. 10. ROC curves for performance evaluation of AdaBoost and SVM.

for RGB images. However, the number of image data used for training was not sufficient, it is necessary to collect more image data to discuss about its ability. This study will be implemented into smartphone in the future works.

\section{References}

(1) World Health Organization: "Visual impairment and blindness", Fact sheets, Media centre, 2014 http://www.who.int/mediacentre/factsheets/fs282/en/ (accessed 12/06/2017)

(2) N. Abe, N. Hashimoto: "Walking Accident National Survey to Maintain Visually Handicapped Persons Walking Environment", The bulletin of Hachinohe Institute of Technology, No.24, pp.81-92, 2005 (in Japanese)

(3) API Co. Ltd.: "Smart Electronics White Cane System", http://www.api-kk.com/denshi-hakujo/ (accessed 12/06/2017)

(4) S. Wang, H. Pan, et al.: "RGB-D Image-Based Detection of Stairs, Pedestrian Crosswalks and Traffic Signs", Journal of Visual Communication Image Representation, No.25, pp.263-272, 2014

(5) S. Murakami, M. Shimakawa, K. Kiyota, T. Kato: "Study on Stairs Detection using RGB-Depth Images", Joint Conference of 7 th SCIS and 15th ISIS (SCIS\&ISIS2014), TP5-3-3-(1), 2014

(6) M. Shimakawa, S. Murakami, K. Kiyota: "Study on Stairs Detection using RGB-Depth Images", The 31st Fuzzy System Symposium, FB2-1, pp.699-702, 2015 (in Japanese)

(7) R. Akutagawa, M. Shimakawa, K. Kiyota: "A Study on Staircase Detection by Machine Learning using OpenCV", The 32nd Fuzzy System Symposium, WF14, pp.83-86, 2016 (in Japanese) 